\title{
STUDIES OF OPTICAL ASYMMETRIES IN THE ELETTRA STORAGE RING
}

\author{
O.Ferrando, E.Karantzoulis \\ Sincrotrone Trieste, 34012 Trieste, Italy
}

\begin{abstract}
Studies concerning the influence of linear and non linear imperfections in the ELETTRA Storage Ring are presented. Tracking simulations show that field errors and orbit errors in sextupoles perturb the machine periodicity with a consequent reduction in the dynamic aperture, injection efficiency and lifetime. To improve the machine performances it is important to determine the actual storage ring optics and the perturbation of the periodicity. A useful method to analyze perturbed optics is by measuring and fitting orbit response matrix data. This method allows to determine the magnet gradient distribution and, by adjusting the quadrupole field strengths, it is possible to restore the periodicity of the lattice. At ELETTRA we have analyzed measured response matrices and reconstructed the real optics of the machine. The results of the analysis are presented in this paper.
\end{abstract}

\section{INTRODUCTION}

ELETTRA is a $2.0 \mathrm{GeV}(2.4 \mathrm{GeV}$ at $5 \%$ of the user time) third generation synchrotron light source. The lattice is of the expanded Chasman-Green type with vertical and horizontal tunes of 8.2 and 14.3. The beta functions, shown in Figure 1 together with dispersion are $8.2 \mathrm{~m}$ horizontally and $2.6 \mathrm{~m}$ vertically in the center of the dispersive free straight sections. The emittance is 7 $\mathrm{nm}$ rad at $2.0 \mathrm{GeV}$. The maximum horizontal dispersion is $0.4 \mathrm{~m}$. During commissioning of the Storage Ring in October 1994 a first measurement was performed to determine optical asymmetries in the lattice. The techniques used were post-processing of the measured response matrices as well as correlating tune shifts to quadrupole setting variations. For the analysis of the sensitivity matrices a computer program was developed [1] that minimized the $\chi^{2}$ difference between the model and the measured response matrix varying the individual quadrupole strengths and using a non linear method of minimization called simplex multidimensional minimization. It analyzed beta beats, beta functions and phase advance and it was also able to correct the optics to the nominal one. Both asymmetry measurements gave a beta beating in the lattice of $20 \%$ horizontally and $10 \%$ vertically.

The work presented in this paper is a more complete analysis of the problems related to optical asymmetries. We use a linear perturbation approach in which the model response matrix is expanded to first order in quadrupole strengths.

The goal of this study is to construct a self-consistent first order optics model of ELETTRA which gives us detailed information about beta functions, dispersion, gra-

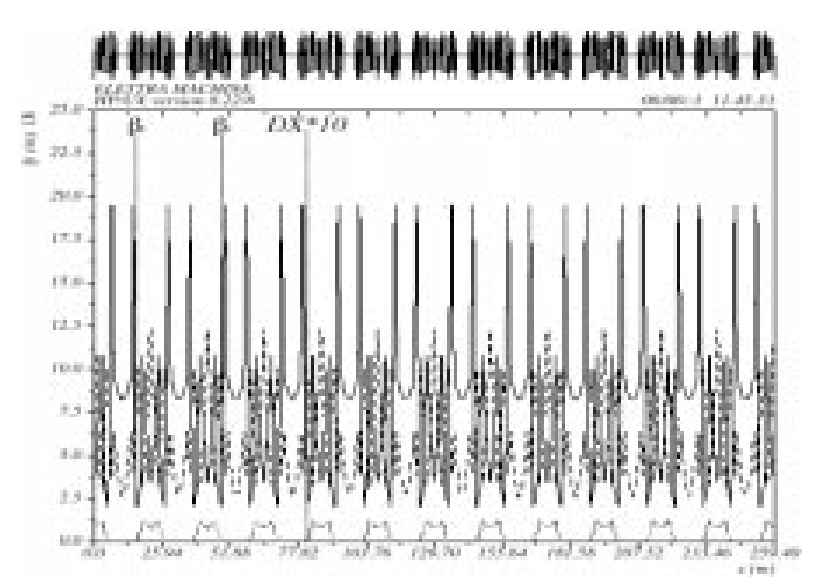

Figure 1: Linear optics of ELETTRA

dient errors, corrector strength errors and beam position monitor linearity factors.

\section{THE METHOD}

The method used to investigate optical asymmetries in the lattice is based on linear analysis of the measured orbit response matrix data [2]. A measured orbit response matrix $\mathbf{M}_{\text {measured }}$ is generated by changing the steering magnet excitation and measuring the orbit change with beam position monitors (BPMs). Mathematically, the orbit response matrix $\mathrm{M}$ is defined by the relation between the $\mathrm{x}, \mathrm{y}$ orbit perturbations at BPMs and the $\theta_{\mathrm{x}}, \theta_{\mathrm{y}}$ changes in steering magnet strengths:

$$
\left(\begin{array}{l}
\mathbf{x} \\
\mathbf{y}
\end{array}\right)=\mathbf{M}\left(\begin{array}{l}
\theta \mathbf{x} \\
\theta \mathbf{y}
\end{array}\right)
$$

It is also possible to generate a model response matrix $\mathrm{M}_{\text {model }}$ assuming a gradient distribution in the storage ring and using an accelerator optics program to calculate the model. The accelerator program used in our case is MAD [3]. The actual gradient distribution of the machine can be determined by varying a set of parameters in the model and minimizing the $\chi^{2}$ deviation between $\mathrm{M}_{\text {measured }}$ and $\mathrm{M}_{\text {model }}$ The parameters changed in the MAD model are the quadrupole fields and the bending gradients since the bending magnets have a gradient component for vertical focusing.

There are $96 \times 2$ BPMs and $82 \times 2$ steering magnets, so there are 31488 elements in the fully coupled matrix and 15744 elements in the uncoupled matrix. For the analysis of the perturbed beta functions we have developed a computer program GRAFIT (Gradient Fit) that varies the gradients and minimizes the $\chi^{2}$ difference between the measured matrix and the model using the method of SVD (singular value decomposition). Since actually the response matrix is not a linear function of the gradients, the program must be iterated until it converges to the 
minimum chi-square. For the analysis of the horizontal and vertical dispersion we have used the program LOCO (Linear Optics from Closed Orbits) [4]. The response matrix has been measured with the program GLOC (Global and Local Orbit Correction) [5].

\subsection{Quadrupole strength analysis}

The storage ring has three groups of quadrupoles: 24 long (Q2), 24 medium (QF), 60 short (24 Q1, 24 Q3, 12 $\mathrm{QD})$. For the fit we have varied in the optics model individually quadrupole and bending strengths. Table 1 reports the average quadrupole strengths obtained for each group, the rms and peak to peak variations.

Table 1 : Fit quadrupole strengths

\begin{tabular}{|l|l|l|l|}
\hline Quadrupoles & $\begin{array}{l}<\mathrm{K}> \\
\mathrm{m}^{-2}\end{array}$ & $\begin{array}{l}\text { rms } \\
\text { deviation } \\
(\%)\end{array}$ & $\begin{array}{l}\text { peak to } \\
\text { peak (\%) }\end{array}$ \\
\hline Q1 & -1.9322 & \pm 0.96 & \pm 2.35 \\
\hline Q2 & 2.2423 & \pm 0.21 & \pm 0.37 \\
\hline Q3 & -1.3321 & \pm 1.56 & \pm 3.5 \\
\hline QD & -1.3864 & \pm 0.76 & \pm 1.25 \\
\hline QF & 2.2236 & \pm 0.35 & \pm 0.6 \\
\hline
\end{tabular}

To evaluate the accuracy of the analysis method ten orbit response matrices have been analyzed and we have found that the variation in the gradient fit between the different data sets is about $0.12 \%$.

The response matrices analysis has been repeated using also the program LOCO to have a comparison with the results obtained from our program. We have found that the gradient distribution obtained with the two programs differ by about $0.15 \%$. The difference is due to the fact that in the LOCO program it is possible to vary also the corrector strengths and the BPM gains thus increasing the accuracy of the fit. The rms variations in the quadrupole groups are particularly large compared with the available magnetic measurement since they are not merely due to calibration errors but they take into account the effects of longitudinal magnet misalignments, quadrupoles and bending magnet tilts and orbit errors in sextupoles. To increase the accuracy of the gradient fit it is necessary to insert in the MAD model used to calculate $\mathrm{M}_{\text {model }}$ the actual rotation magnet angles and the longitudinal misalignments. We have not yet included in our model a realistic distribution of alignment errors since at present only partial survey data is available on transverse alignment errors but not about the rotation angles of the magnets. In autumn of this year more precise survey measurement will be done using a laser-tracker system for the realignment of the machine. Then we can insert in our model the actual error distribution and evaluate the influence on the storage ring optics. Using the individual quadrupole gradients determined by the fit, it is possible to compute the beta functions. The perturbed optics is shown in Figure 2.

The Figures 3 and 4 show the horizontal and vertical

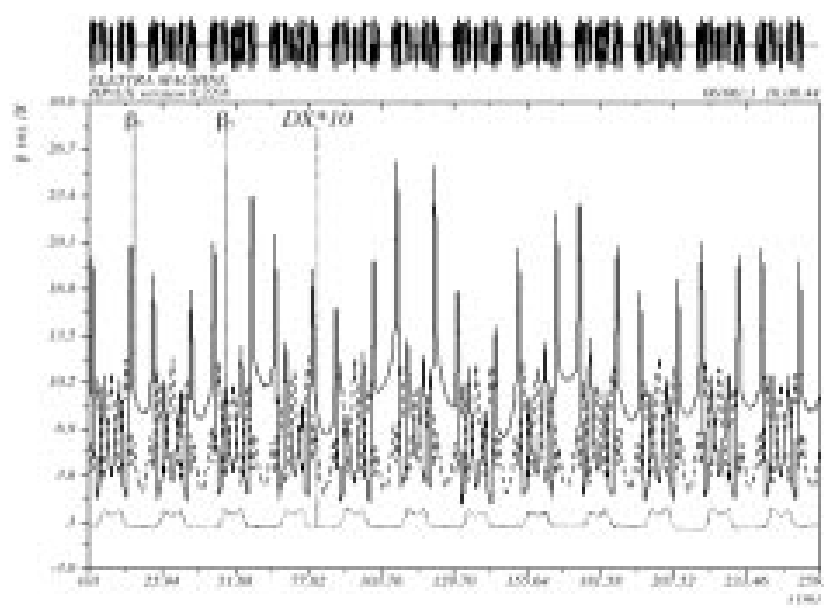

Figure 2: Perturbed Optics

beta-beat. The maximum variations of the beta functions from the model are $33 \%$ horizontally and $22 \%$ vertically, while the rms perturbations are $1.32 \mathrm{~m}$ in the horizontal plane and $0.68 \mathrm{~m}$ in the vertical plane. The betatron tunes of the fit model agree with the measured tunes within the accuracy of the measurement.

Optics simulations show that such beta beating can be easily achieved by including in the MAD model optics the actual magnet field errors available from magnetic measurements and generated error distributions with the following rms values: $\Delta x=\Delta y=\Delta s=0.12 \mathrm{~mm}$ and $\Delta \varphi=\Delta \psi=\Delta \theta=30 \mu \mathrm{rad}$. From the simulation data we have evaluated that the contribution on the beta beat from orbit errors in sextupoles is about $19 \%$ horizontally and $7 \%$ vertically.

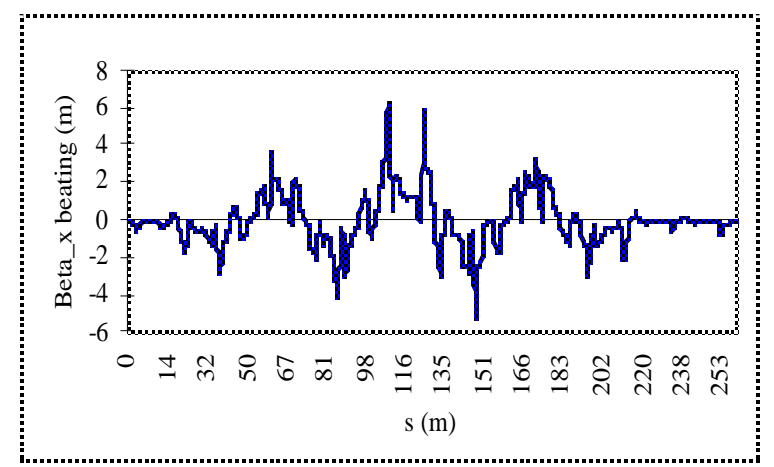

Figure 3: Horizontal beta beating

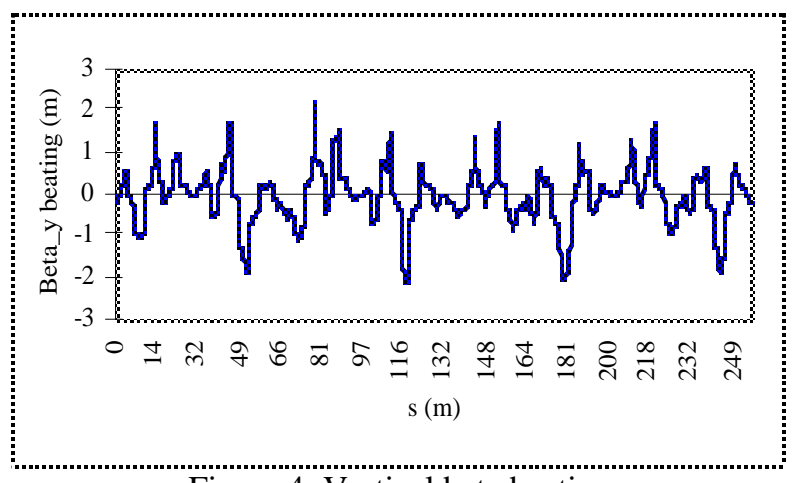

Figure 4: Vertical beta beating 


\subsection{Dispersion analysis}

The analysis of the response matrix gives also detailed information about the horizontal and vertical dispersion.

The calculated dispersion includes the gradient errors obtained from the response matrix analysis and the dipole errors which come from variations of dipole magnet strengths. As shown in Figure 5 and 6 the fitted dispersion overlap very well the measured dispersion for both the horizontal and vertical planes. A small discrepancy between the fit and measured horizontal dispersion $(0.16 \%$ rms value) is due to the fact that we have neglected in the model the dipole kicks generated by the transverse quadrupole misalignments. The agreement of the measured and fitted dispersion confirms the correctness of the used analysis method.

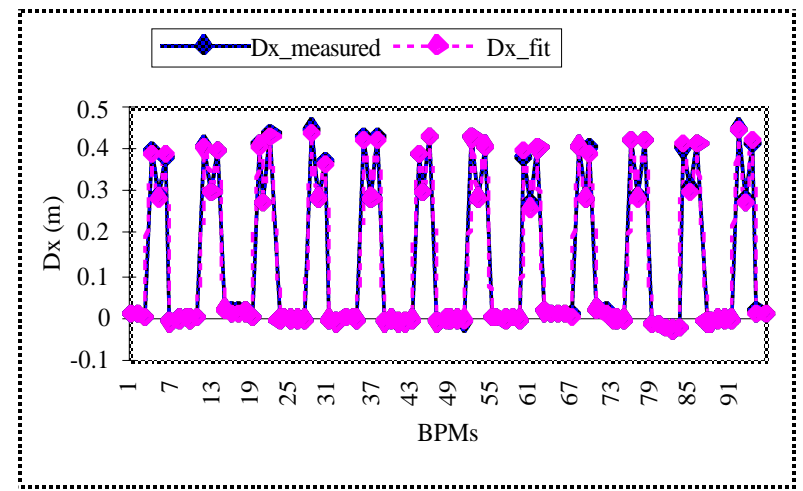

Figure 5: Measured and calculated horizontal dispersion

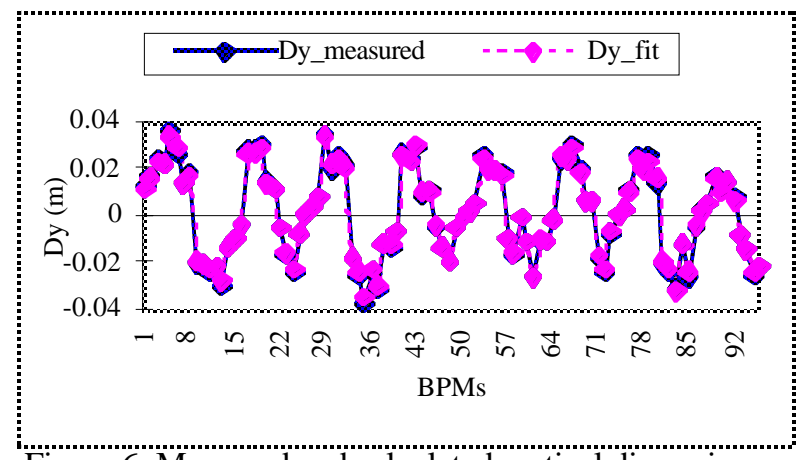

Figure 6: Measured and calculated vertical dispersion

\section{CONCLUSION}

Analysis of the measured orbit response matrix is a powerful way to get information concerning the storage ring optics.

From this study we have been able to reconstruct the actual beta functions of the machine. We have determined the gradient variations of quadrupoles in each group and we have seen that the beta beating obtained in the past (20\% to $10 \%$ ) are now incremented to $33 \%$ and $22 \%$ for the horizontal and vertical plane respectively. From optics simulations we have evaluated that the beta beating can be partially attributed to quadrupoles and bending magnet tilts and longitudinal misalignments and partially to orbit errors in sextupoles. The dispersion and tune measurements show very good agreement with the calibrated model even if the model takes into consideration only gradient and dipole errors. This confirms that the parameter values obtained with the linear response matrix analysis accurately predict the true storage ring.

As pointed out in section 2.1, when a complete set of survey data is available, more investigations will be performed to analyze the effects of quadrupole and bending magnets tilts and longitudinal misalignments on the beta beat. It will be also possible to get more precise information on orbit errors in sextupoles, corrector strength errors and BPMs gain factors. Once the analysis is completed the final goal of restoring the periodicity of the machine will be performed.

\section{ACKNOWLEDGEMENTS}

The author would like to thank J.Safranek for use of his LOCO program.

\section{REFERENCES}

[1] E. Karantzoulis, Optiks: An Optics Measurement and Correction Program for ELETTRA, European Particle Accelerator Conference, London 1994.

[2] W.J. Corbett and V. Ziemann, in Proceedings of the 1993 Particle Accelerator Conference, Washington, p.108

[3] H.Grote, F.C.Iselin, The MAD Program, Version 8.22. CERN/SL/90-13(AP)

[4] J.Safranek, Nucl.Inst.and Meth. A388, 27 (1995)

[5] E. Karantzoulis, Orbit Control Advances at ELETTRA, Procs. EPAC 2000, Vienna, June 2000 